\title{
CAF vs. CMF Combination Chemotherapy in Advanced Breast Cancer Patients
}

\author{
Dr. R. Navajothi ${ }^{1}$, Dr. R. Sudha ${ }^{2}$ \\ ${ }^{1}$ Associate Professor, Department of Pharmacology Govt. Sivagangai Medical College, Sivagangai, Tamilnadu, India \\ ${ }^{2}$ AssociateProfessor, Department of Pharmacology, KAPV Govt Medical College, Tiruchirappalli, Tamilnadu, India
}

\begin{abstract}
Breast cancer is the second most common neoplasm in women. There has been a modest increase in the duration of survival of most women with breast cancer. This increase in survival has come about because of the recognition that breast cancer is a systemic disease. Systemic nature is the driving force behind newer chemotherapeutic approaches. The purpose of this study was to determine the

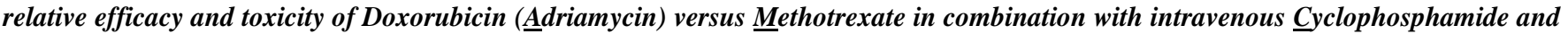
5-Fluorouracil as a palliative therapy in locally advanced, locoregionally recurrent and distant metastatic breast cancer patients.The study was carried out in a tertiary care centre, for a period of 20 months. Histopathologically confirmed breast cancer patients (female) with ductal carcinoma or lobular carcinoma who had locoregionally advanced disease were enrolled in the study. Twenty eight patients received CAFregimen and twenty patients received CMF regimen with prophylactic antiemetics after baseline biochemical and metastasis evaluation. Acute toxicity was evaluated for 24 hours on the day of chemotherapy and followed up for the side effects. Patients were planned for treatment with minimum of 4 cycles of chemotherapy. The response was quantified as complete response, partial response and no response. Complete responders were given 2 more cycles and treatment was stopped. Non responders were withdrawn from the study. Partial responders were planned for 2 more cycles and reassessed and chemotherapy was continued till progressive disease was seen or upto a maximum of 9 cycles whichever occurred earlier. Results were analysed by Students' $t$ test. Complete response rate in CAF arm was $39 \%(11 / 28)$ \& in CMF arm was 15\%(3/20). Overall response (complete+ partial) rate in CAF arm was $64 \%(18 / 28)$ \& in CMF arm was 55\%(11/20). Both CAF and CMF regimen were active,safe \& convenient.
\end{abstract}

Keywords: CAF, CMF, Complete response, Partial response

\section{Introduction}

Breast cancer is the second most common neoplasm in women affecting $18 \%$ worldwide.9,00,000 new cases are diagnosed and 5,19,000 deaths are occurring every year. In India incidence is 20 per $1,00,000$ population. Germ cell mutation is associated with $10 \%$ of all breast cancers while other 90\% occur sporadically. BRCA1 Associated breast cancer occurring in young women has aggressive features and characterized by a triple negative phenotype(ER,PR \& HER- 2 -negative). The HER-2/neu, oncogene's amplification is a significant predictor of lower survival. Reduced expression of a putative antimetastatic gene, Nm23is a potentially important prognostic factor ${ }^{1}$

Increased use of screening mammography has nearly doubled the frequency of in situ breast cancer (stage 0 diagnosis ${ }^{2}$. There has been a modest increase in the duration of survival of most women with breast cancer. This increase in survival has come about because of the recognition that breast cancer is a systemic disease. Systemic nature is the driving force behind newer chemotherapeutic approaches. Multi drug trials increased the survival in modern breast cancer treatment ${ }^{3}$

In the multimodal treatment for metastatic breast cancer, the preferred combination regimen by NCCN (National Comprehensive Cancer Network) guidelines is CAF. Weekly high dose 24- hour infusion of 5-flurouracil in combination with folinic acid or paclitaxel or both appear promising. 4 Incorporation of taxanes have become cornerstone of modern chemotherapy. Only a small percentage of patients with metastatic breast cancer achieve long term disease free survival. Although the mortality rates for breast cancer patients have improved over the last decade, the loss of 40,000 lives each year as a result of metastasis has remained constant.

\section{Materials \& Method}

The study was conducted in department of Medical Oncology, Govt. Rajaji Hospital, Madurai for 20 months after getting approval from institutional ethical committee. Forty eight female patients with advanced breast cancer from southern tamil nadu were enrolled in the study after getting informed consent. Histopathologically confirmed breast cancer patients with ductal carcinoma or lobular carcinoma who had any of the following (measurable recurrent or metastatic disease after primary surgery, locoregionally advanced disease with or without distant metastasis fitting into the stage of IIIA/IIIB/IV, measurable recurrent or metastatic disease after adjuvant CMF chemotherapy will be enrolled in the CAF treatment arm \& measurable recurrent or metastatic disease after adjuvant CAF chemotherapy will be enrolled in the CMF treatment arm) were included in the study.

Histopathologically confirmed breast cancer patients with ductal carcinoma or lobular carcinoma who had any of the following (locoregional disease amenable for palliative resection or irradiation, extensive prior treatment with several chemotherapy regimens, Impaired renal or hepatic function unless the functional abnormality was due to metastatic involvement, Impaired bone marrow function with anemia ( $\mathrm{Hb}<9 \mathrm{gm}$ ), leucopaenia (WBC <4,000), thrombocytopaenia (Platelets $<1,00,000$ ) unless dysfunction was due to metastatic involvement)were excluded from the study. 


\section{International Journal of Science and Research (IJSR) ISSN (Online): 2319-7064 \\ Index Copernicus Value (2013): 6.14 | Impact Factor (2015): 6.391}

Twenty eight patients received CAFregimen and twenty patients received CMF regimen with prophylactic antiemetics after baseline biochemical evaluation and evaluation for metastasis. Acute toxicity was evaluated for 24 hours on the day of chemotherapy and further followed up for the other side effects /toxicity. Patients were planned for treatment with minimum of 4 cycles of chemotherapy with intercycle follow-up for 20 days. Pre chemotherapy evaluation was done before each cycle for assessing toxicity. The response assessment was done before each cycle and formal assessment done prior to $5^{\text {th }}$ cycle.

Response was quantified as(i) complete response(complete regression of disease in tumor sites lasting at least 4 weeks),(ii) partial response(50\% or greater reduction in the size of the tumor lasting at least 4 weeks \& without appearance of any new lesions during treatment) and (iii) no response(any regression in the tumor which is less than $50 \%$ of the original size and any increase in the tumor which is not greater than $25 \%$ of the original size).

After completing 4 cycles, complete responders were given 2 more cycles and treatment was stopped. Non responders were withdrawn from the study and offered alternative treatment. Partial responders were planned for 2 more cycles and reassessed and chemotherapy was continued till progressive disease was seen or up to a maximum of 9 cycles whichever occurred earlier. Results were analyzed with students 't test.

\section{Results}

In the present study twenty eight patients were enrolled in CAFregimen and twenty patients were enrolled in CMF regimen. No patients were lost to follow up. Complete Response rate in CAF arm was 39\% (11/28). Complete
Response rate in CMF arm was 15\% (3/20). Though CAF appears superior to CMF there is no statistical significance. Mean duration of complete response in the CAF arm was 5(2-18) months.. Mean duration of complete response in the CMF arm was 3(2-4) months. Overall response (complete+ partial) rate in CAF arm was 64\%(18 / 28) \& in CMF arm was $55 \%(11 / 20)$. (Figure-1)

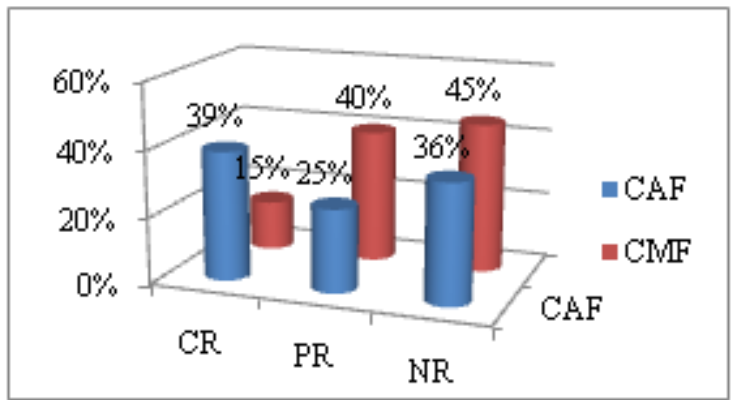

Figure 1: Response rate to CAF \&CMF

The response pattern to the two arms in relation to the Menopausal status (Table-1) and site wise analysis (Table$2 \& 3$ ) also proved superiority of CAF over CMF regimen. Liver metastasis showed better response.

Table 1: Menopausal status wise Response to CAF \& CMF

\begin{tabular}{|l|l|l|l|}
\hline \multicolumn{2}{|c|}{$\begin{array}{c}\text { Menopausal } \\
\text { Status }\end{array}$} & Premenopausal & Postmenopausal \\
\hline \multirow{4}{*}{ CAF } & NO & 15 & 13 \\
\cline { 2 - 4 } & CR & $5(33 \%)$ & $5(38 \%)$ \\
\cline { 2 - 4 } & PR & $4(27 \%)$ & $4(31 \%)$ \\
\cline { 2 - 4 } & OR & $9(60 \%)$ & $9(69 \%)$ \\
\hline \multirow{5}{*}{ CMF } & NO & 8 & 12 \\
\cline { 2 - 4 } & CR & $1(13 \%)$ & $2(17 \%)$ \\
\cline { 2 - 4 } & PR & $3(37 \%)$ & $5(41 \%)$ \\
\cline { 2 - 4 } & OR & $4(50 \%)$ & $7(58 \%)$ \\
\hline
\end{tabular}

Table 2: Sitewise Complete Response to CAF \& CMF

\begin{tabular}{|c|c|c|c|c|c|c|}
\hline \multirow{2}{*}{ Sites } & \multicolumn{3}{|c|}{ CAF } & \multicolumn{3}{c|}{ CMF } \\
\cline { 2 - 7 } & NO & CR & Mean \pm SE & NO & CR & Mean \pm SE \\
\hline Chest wall recurrence & 14 & $4(29 \%)$ & $0.29 \pm 0.17$ & 13 & $2(15 \%)$ & $0.15 \pm 0.17$ \\
\hline Lymphnodes & 17 & $7(41 \%)$ & $0.41 \pm 0.14$ & 18 & $3(17 \%)$ & $0.17 \pm 0.14$ \\
\hline Breastmass & 4 & 0 & 0 & 1 & 0 & 0 \\
\hline Liver & 4 & $2(50 \%)$ & $0.5 \pm 0.35$ & 3 & 0 & $0 \pm 0.35$ \\
\hline Bone & 4 & $2(50 \%)$ & $0.5 \pm 0.55$ & 1 & 0 & $0 \pm 0.55$ \\
\hline Pleura & 5 & $1(20 \%)$ & 0 & NIL & NIL & NIL \\
\hline OVERALL & 28 & $11(39 \%)$ & $0.39 \pm 0.13$ & 20 & $3(15 \%)$ & $0.15 \pm 0.13$ \\
\hline
\end{tabular}

Table 3: Sitewise Overall Response to CAF \& CMF

\begin{tabular}{|c|c|c|c|c|c|c|}
\hline \multirow{2}{*}{ Sites } & \multicolumn{4}{|c|}{ CAF } & \multicolumn{4}{c|}{ CMF } \\
\cline { 2 - 7 } & NO & OR & Mean \pm SE & NO & OR & Mean \pm SE \\
\hline $\begin{array}{c}\text { Chest wall } \\
\text { recurrence }\end{array}$ & 14 & $7(50 \%)$ & $0.5 \pm 0.2$ & 13 & $6(46 \%)$ & $0.46 \pm 0.2$ \\
\hline Lymphnodes & 17 & $13(70 \%)$ & $0.76 \pm 0.17$ & 18 & $9(50 \%)$ & $0.5 \pm 0.17$ \\
\hline Breastmass & 4 & $2(50 \%)$ & $0.5 \pm 0.55$ & 1 & 0 & $0 \pm 0.55$ \\
\hline Liver & 4 & $3(75 \%)$ & $0.75 \pm 0.35$ & 3 & $2(67 \%)$ & $0.66 \pm 0.35$ \\
\hline Bone & 4 & $3(75 \%)$ & $0.75 \pm 0.55$ & 1 & 0 & $0 \pm 0.55$ \\
\hline Pleura & 5 & $1(20 \%)$ & 0 & NIL & NIL & NIL \\
\hline OVERALL & 28 & $18(64 \%)$ & $0.64 \pm 0.6$ & 20 & $11(55 \%)$ & $0.55 \pm 0.6$ \\
\hline
\end{tabular}

Total cycles of CAF\& CMF administered were 162 \&109. Extravasation injury was nil in CMFarm \& 14\% in CAFarm. Emesis was mild (60\%) in CMF arm. Emesis was mild in

24(72\%) patients, moderate in 4 (14\%) patients \& severe in $4(14 \%)$ patients of CAF arm. Mucositis was mild (60\%) in CMF arm. Mucositis was mild in $4(14 \%)$ patients, moderate in $4(14 \%)$ patients \& severe in 6 (21\%) patients of CAF arm. Mild alopecia(<25\%hair loss) was seen in all patients who received CMF arm. In CAF arm all patients had severe alopecia (>50\%hair loss). Nadir neutropaenia (WBC $<1000 /$ cu.mm) was $14 \%$ in CAF arm \& nil in CMF arm. There was no chemotherapy related mortality in both arms.

\section{Discussion}

\section{Volume 5 Issue 4, April 2016} www.ijsr.net 


\section{International Journal of Science and Research (IJSR) \\ ISSN (Online): 2319-7064 \\ Index Copernicus Value (2013): 6.14 | Impact Factor (2015): 6.391}

The purpose of this study was to determine the relative efficacy and toxicity of doxorubicin versus methotrexate in combination with intravenous cyclophosphamide and 5fluorouracil as a palliative therapy in locally advanced, locoregionally recurrent and distant metastatic breast cancer patients. Worldwide, numerous clinical trials ${ }^{5,6,7,8}$ had established the similar objective response to both regimens. Superiority of CAF regimen was established by Martin ${ }^{9}$. The overview analysis of polychemotherapy analyzed results from 17 trials that directly compared CAF with CMF and demonstrated a significant advantage with CAF regimen ${ }^{10 .}$ In the present study if the same response pattern were to be confirmed in a larger patients the statistical analysis may well demonstrate that CAF protocol is indeed superior to CMF protocol in terms of producing higher rates of complete response and overall response.

Best responses were seen with subcutaneous recurrences in anterior chest wall and worst responses in bone metastasis similar to study conducted by Smalley ${ }^{11}$. At the commencement of study there was a certain amount of anxiety with regard to the anticipated toxicity of CAF regimen in our patients. Although the clinical toxicity of $\mathrm{CAF}$ is greater than that of CMF, the levels were manageable and clinically acceptable in our patients similar to the study by Martin.

\section{Conclusion}

Metastatic breast cancer is usually incurable. The aim of combination chemotherapy in palliative metastatic setting is to improve symptoms, quality of life and extend survival. It is important to choose therapy with optimal activity while minimizing toxicity. The least toxic approach is preferred when efficacy is considered equal. Combination chemotherapy is associated with increased responses compared with single-agent chemotherapy. However, treatment using single agents in a sequential fashion is associated with less toxicity than the use of a combination regimen. Chemotherapy is considered if there is a short disease free interval, involvement of vital organs and tumour is hormone receptor negative. Hormonal therapy is considered if there is a long disease free interval and tumour is hormone receptor positive. Immunotherapy by HER-2 targeted agents increase rate and duration of response with HER2 over expressing tumors ${ }^{12}$.Identifying those patients who may be benefitted by undergoing treatment can be difficult because there is little evidence to provide directions to the clinicians ${ }^{13}$.

Patients should be encouraged to participate in clinical trials of novel agents in endocrine therapy and biologic therapy. The importance of quality of life in the treatment is reflected by the increasing number of recent clinical trials that incorporate measures of quality of life as end points of study

${ }^{14}$. In this study both CAF \&CMF regimens were active, safe and convenient. Clinically CAF regimen is better than CMF though statistically not significant. This may be due to relatively small sampling of patients and an extended study in large population is needed. More toxicity was anticipated in CAF regimen but patients sailed through their cycles without undue myelosuppression.

\section{Limitations of the study}

Large sample size \& extended duration of the study are warranted

\section{Conflict of interest}

Conflict of interest declared none

\section{Acknowledgement}

I am thankful to my director Dr. S. Kanagambal \& Dr. M. K. Muthukumaraswamy, Head of the Department of Medical Oncology.

\section{References}

[1] Hennessy. C.,et al. Expression of the antimetastatic gene nm23 in human breast cancer:an association with good prognosis.Journal of the national cancer institute, 1991;83:281-5

[2] Osteen.RT.,et al. Breast cancer:National cancer database: Annual review of patient care1994. Atlanta, GA, The american cancer society,pp 56-71

[3] Fisher.B.,et al. The contribution of recent clinical trials of primary breast cancer therapy to an understanding of tumor biology.Cancer 1980 ;46:1009-1025.

[4] Klaassen. U., et al. Flurouracil -based combinations in the treatment for metastatic breast cancer. Oncology- Huntingt, 1998 ; 12 (1 supple1): $31-5$

[5] Cocconi.G.,et al. Chemotherapy versus combination of chemotherapy and endocrine therapy in advanced breast cancer. A prospective randomized study. Cancer ,1983; 51:581-588

[6] Mouridsen.HT.,et al.Combined cytotoxic and endocrine therapy in postmenopausal patients with advanced breast cancer. A randomized study of CMF vs CMF plus tamoxifen. Eur J Cancer Clinical Oncology, 1985; 21:291-299

[7] .Nomura.Y. Adriamycin -breast cancer. Gan -To-KagakuRyoho,1996 ; 23(14):1911-5

[8] Perry. MC.,et al. Chemohormonal therapy in advanced breast cancer :Cancer and Leukemia Group B Protocol 8081. J Clini Oncol ,1987; 5:1534-1545

[9] Martin.M.,et al. Doxorubicin in combination with fluorouracil and cyclophosphamide versus methotrexate in combination with fluorouracil and cyclophosphamide as palliative chemotherapy for metastatic breast cancer: a study by the GEICAM group (Spanish Breast Cancer Research Group), Spain.Ann Onco, 2003 Jun ;14(6):833-42.

[10] Effects of chemotherapy and hormonal therapy for recurrence in breast cancer: An overview of randomized trials. Lancet 2005;365:1687-1717

[11] Smalley.RV., Lefonto.J.,Bartolucci. A.,et al. A comparison of cyclo,doxo \& 5-FU and cyclo,metho\& 5-FU in patients with advanced breast cancer; breast cancer Res Treat, 1983; 3:209

[12] PICCART-GEBHART. MJ., etal. Trastuzumab in breast cancer patients with overexpression of HER2 oncogene.N. Engl.J Med 2005;353:1659-1672

[13] Anders CK, Peppercorn J. Treating in the dark: Unanswered questions oncosts and benefits of late line therapy for metastatic breast cancer. Cancer Investig 2009;27:13-16.

[14] Osoba D, Slamon DJ, Burchmore M et al. Effects on quality of life of combined Trastuzumab and chemotherapy in women with metastatic breast cancer. J Clin Oncol 2002;20:31063113 